\title{
Branches of forced oscillations for a class of constrained ODEs: a topological approach
}

\author{
Alessandro Calamai and Marco Spadini
}

\begin{abstract}
We apply topological methods to obtain global continuation results for harmonic solutions of some periodically perturbed ordinary differential equations on a $k$-dimensional differentiable manifold $M \subseteq$ $\mathbb{R}^{m}$. We assume that $M$ is globally defined as the zero set of a smooth map and, as a first step, we determine a formula which reduces the computation of the degree of a tangent vector field on $M$ to the Brouwer degree of a suitable map in $\mathbb{R}^{m}$. As further applications, we study the set of harmonic solutions to periodic semi-explicit differential-algebraic equations.
\end{abstract}

Mathematics Subject Classification (2000). 34C40, 34A09, 34C25.

Keywords. Ordinary differential equations on manifolds, Differential algebraic equations, Degree of a vector field, Periodic solution.

\section{Introduction and preliminaries}

In this paper we study $T$-periodic solutions of some parametrized families of $T$-periodic constrained ordinary differential equations (ODEs). More precisely, we study periodically perturbed autonomous ODEs on a differentiable submanifold of some Euclidean space, under the assumption that such a manifold is globally defined as the zero set of a smooth map. We consider the two different cases of nontrivial unperturbed equation and of perturbation of the zero vector field. We adopt a topological approach and we make use of results which are based on the fixed point index. However, our techniques require just the notion of the degree (often called characteristic or rotation) of tangent vector fields to differentiable manifolds, which in the 'flat' case, that is when the manifold is an open subset of an Euclidean space, is essentially the well known Brouwer degree. As an application of our results, we study $T$-periodic solutions of particular parametrized differential-algebraic equations (DAEs), for which we will prove global continuation results. 
Recently, DAEs have received an increasing interest due, in particular, to applications in engineering and have been the subject of extensive study (see e.g. [9] for a comprehensive treatment) aimed mostly (but not only) to numerical methods. Our approach here, inspired by [1] and [11], is directed towards qualitative theory of some particular DAEs which are studied by means of topological methods, making use of the equivalence of the given equations and suitable ODEs on manifolds. Relatively to $[1,11]$, here we operate a change of perspective: assuming the viewpoint of ODEs on manifold allows us to present the matter in a general and extensively studied framework (see e.g. [4]).

Our first goal is to obtain a formula for the computation of the degree of tangent fields to a $k$-dimensional differentiable submanifold $M$ of $\mathbb{R}^{m}$, in the particular case when the manifold is defined implicitly as the zero set of a smooth function $g: U \rightarrow \mathbb{R}^{s}$, with $s=m-k$ and $U \subseteq \mathbb{R}^{m}$ open and connected, and assuming that with an appropriate choice of (orthonormal) coordinates one can decompose $\mathbb{R}^{m}$ as $\mathbb{R}^{k} \times \mathbb{R}^{s}$ in such a way that the Jacobian matrix of $g$ with respect to the last $k$ variables, $\partial_{2} g(x, y)$, is nonsingular for all $(x, y) \in U$. Notice that, in this case, $0 \in \mathbb{R}^{s}$ is a regular value of $g$ so that $M=g^{-1}(0)$ is a smooth submanifold of $\mathbb{R}^{m}=\mathbb{R}^{k} \times \mathbb{R}^{s}$ of codimension $s$.

The formula we find (see Theorem 4.1 below) reduces the computation of the degree of a tangent vector field on $M$ to that of an appropriate map in $\mathbb{R}^{m}$. More precisely, let $\varphi: M \rightarrow \mathbb{R}^{m}$ be tangent to $M$, in the sense that $\varphi(\xi)$ belongs to the tangent space $T_{\xi} M$ of $M$ at $\xi$ for any $\xi \in M$. Let also $\widetilde{\varphi}$ be any extension of $\varphi$ to $U$. With a small abuse of notation, we will write, according to the above decomposition,

$$
\widetilde{\varphi}(\xi)=\widetilde{\varphi}(x, y)=\left(\widetilde{\varphi}_{1}(x, y), \widetilde{\varphi}_{2}(x, y)\right),
$$

and define $\mathcal{F}: U \rightarrow \mathbb{R}^{m}$ as $\mathcal{F}(x, y)=\left(\widetilde{\varphi}_{1}(x, y), g(x, y)\right)$, for any $(x, y) \in U$. We will prove that

$$
\operatorname{deg}(\varphi, M)=\mathfrak{s} \operatorname{deg}(\mathcal{F}, U)
$$

where $\mathfrak{s}$ is the (constant) sign of $\operatorname{det}\left(\partial_{2} g\right)$ on the connected set $U$. Observe that the just defined vector field $\mathcal{F}$ on $U$ may well not be tangent to $M$. In fact, on $M$, the second component of $\mathcal{F}$ is forced to be zero regardless of the shape of $M$.

The above formula (1.1) is equivalent to a result proved in [11] but we provide here a simplified proof. Notice that (1.1) does not depend on the chosen extension of $\varphi$. Notice also that, since in Euclidean spaces vector fields can be regarded as maps and vice versa, the degree of the vector field $\mathcal{F}$ that appears in the second member of (1.1) is essentially the well known Brouwer degree, with respect to 0 , of $\mathcal{F}$ seen as a map. Hence the degree of $\mathcal{F}$, having a simpler nature than that of $\varphi$, is 'morally' easier to compute.

As an application we study the set of harmonic solutions of the following parametrized differential equations on a manifold $M \subseteq \mathbb{R}^{m}$, with $M=g^{-1}(0)$ and $g$ as above:

$$
\dot{\xi}=f(\xi)+\lambda h(t, \xi), \quad \lambda \geq 0
$$


and

$$
\dot{\xi}=\lambda h(t, \xi), \quad \lambda \geq 0
$$

where $h: \mathbb{R} \times M \rightarrow \mathbb{R}^{m}$ and $f: M \rightarrow \mathbb{R}^{m}$ are continuous maps with the property that $f(\xi)$ and $h(t, \xi)$ belong to $T_{\xi} M$ for any $(t, \xi) \in \mathbb{R} \times M$, and $h$ is $T$-periodic in the first variable.

Notice that locally $M$ can be represented as graph of some map from an open subset of $\mathbb{R}^{k}$ to $\mathbb{R}^{s}$, with $k=m-s$. Thus equations (1.2) can be locally simplified. In view of this fact one might think that it is possible to reduce equations (1.2) to ODEs in $\mathbb{R}^{k}$. It is not so. In fact, globally, $M$ may not be the graph of a map from an open subset of $\mathbb{R}^{k}$ to $\mathbb{R}^{s}$ as, for instance, when $U=\mathbb{R}^{3}$ and $g: \mathbb{R} \times \mathbb{R}^{2} \rightarrow \mathbb{R}^{2}$ is given by

$$
g(x, y)=g\left(x ; y_{1}, y_{2}\right)=\left(e^{y_{1}} \cos y_{2}-x, e^{y_{1}} \sin y_{2}-x\right) .
$$

In this case, although $\operatorname{det} \partial_{2} g(x, y) \neq 0$, one clearly has that the 1-dimensional manifold $M=g^{-1}(0)$ is not the graph of a function $x \mapsto\left(y_{1}(x), y_{2}(x)\right)$. In fact, $M$ consists of infinitely many connected components each lying in a plane $y_{2}=\frac{\pi}{4}+\ell \pi$ for $\ell \in \mathbb{Z}$. (See also Examples 5.2 and 5.8 below.)

Observe also that even when $M$ is a (global) graph of some map $\Gamma$, the expression of $\Gamma$ might be too complicated or impossible to determine analytically, so that the decoupled versions of equations (1.2) may be too difficult to use. A simple example of this fact is obtained by taking $k=s=1, U=\mathbb{R} \times \mathbb{R}$ and $g(x, y)=y^{7}+y-x^{2}+x^{5}$.

As further applications, we will deduce the results of $[1,11]$ about harmonic solutions of periodic semi-explicit DAEs that have either the form

$$
\left\{\begin{array}{l}
\dot{x}=\gamma(x, y)+\lambda \sigma(t, x, y), \quad \lambda \geq 0 \\
g(x, y)=0
\end{array}\right.
$$

or

$$
\left\{\begin{array}{l}
\dot{x}=\lambda \sigma(t, x, y), \quad \lambda \geq 0, \\
g(x, y)=0 .
\end{array}\right.
$$

Here $U \subseteq \mathbb{R}^{k} \times \mathbb{R}^{s}$ is a connected open set, $g: U \rightarrow \mathbb{R}^{s}$ is as above, $\gamma: U \rightarrow \mathbb{R}^{k}$ and $\sigma: \mathbb{R} \times U \rightarrow \mathbb{R}^{k}$ are continuous maps, and $\sigma$ is $T$-periodic in $t$ for a given $T>0$. In fact, as we shall see, equations (1.3a) and (1.3b) are equivalent to (1.2a) and (1.2b), respectively, for appropriate vector fields $f$ and $h$ on the manifold $g^{-1}(0)$. Notice that, as remarked above, although the set $g^{-1}(0)$ is locally the graph of some map of an open set of $\mathbb{R}^{k}$ to $\mathbb{R}^{s}$ so that equations (1.3a) and (1.3b) can be locally decoupled, it is not always possible or convenient to do globally so.

\section{Tangent vector fields and the notion of degree}

We now recall some basic notions about tangent vector fields on manifolds, and introduce the notion of degree of an admissible tangent vector field.

Let $M \subseteq \mathbb{R}^{m}$ be a manifold. Let $w$ be a tangent vector field on $M$, that is, a continuous map $w: M \rightarrow \mathbb{R}^{m}$ with the property that $w(\zeta) \in T_{\zeta} M$ for 
any $\zeta \in M$. If $w$ is (Fréchet) differentiable at $\zeta \in M$ and $w(\zeta)=0$, then the differential $d w_{\zeta}: T_{\zeta} M \rightarrow \mathbb{R}^{m}$ maps $T_{\zeta} M$ into itself (see e.g. [10]), so that the determinant det $d w_{\zeta}$ of $d w_{\zeta}$ is defined. If, in addition, $\zeta$ is a nondegenerate zero (i.e. $d w_{\zeta}: T_{\zeta} M \rightarrow \mathbb{R}^{m}$ is injective) then $\zeta$ is an isolated zero and $\operatorname{det} d w_{\zeta} \neq 0$.

Let $W$ be an open subset of $M$ in which we assume $w$ admissible (for the degree); that is, the set $w^{-1}(0) \cap W$ is compact. Then, one can associate to the pair $(w, W)$ an integer, $\operatorname{deg}(w, W)$, called the degree (or characteristic) of the vector field $w$ in $W$, which, in a sense, counts (algebraically) the zeros of $w$ in $W$ (see e.g. $[5,8,10]$ and references therein). In fact, when the zeros of $w$ are all nondegenerate, then the set $w^{-1}(0) \cap W$ is finite and

$$
\operatorname{deg}(w, W)=\sum_{\zeta \in w^{-1}(0) \cap W} \operatorname{sign} \operatorname{det} d w_{\zeta} .
$$

Observe that in the flat case, i.e. when $M=\mathbb{R}^{m}, \operatorname{deg}(w, W)$ is just the classical Brouwer degree with respect to zero where $V$ is any bounded open neighborhood of $w^{-1}(0) \cap W$ whose closure is contained in $W$.

The notion of degree of an admissible tangent vector field plays a crucial role throughout this paper. It enjoys a number of properties some of which we report here for the sake of future reference.

Additivity. Let $w$ be admissible in $W$. If $W_{1}$ and $W_{2}$ are two disjoint open subsets of $W$ whose union contains $w^{-1}(0) \cap W$, then

$$
\operatorname{deg}(w, W)=\operatorname{deg}\left(w, W_{1}\right)+\operatorname{deg}\left(w, W_{2}\right) .
$$

Homotopy Invariance. Let $h: M \times[0,1] \rightarrow \mathbb{R}^{m}$ be an admissible homotopy (of tangent vector fields) in $W$; that is, $h(\zeta, \lambda) \in T_{\zeta} M$ for all $(\zeta, \lambda) \in M \times[0,1]$ and $h^{-1}(0) \cap W \times[0,1]$ is compact. Then $\operatorname{deg}(h(\cdot, \lambda), W)$ is independent of $\lambda$.

Solution. If $w$ is admissible in $W$ and $\operatorname{deg}(w, W) \neq 0$, then $w$ has a zero in $W$.

The Additivity Property implies the following important property:

Excision. Let $(w, W)$ be admissible. If $V \subseteq W$ is open and contains $w^{-1}(0) \cap W$, then $\operatorname{deg}(w, W)=\operatorname{deg}(w, V)$.

Another property that plays an important role in this paper is the following one which allows the comparison between the degrees of vector fields that correspond under diffeomorphisms. Recall that if $v: N \rightarrow \mathbb{R}^{n}$ and $w: M \rightarrow \mathbb{R}^{m}$ are tangent vector fields on the differentiable manifolds $N \subseteq \mathbb{R}^{n}$ and $M \subseteq \mathbb{R}^{m}$, and if $\rho: W \rightarrow V$ is a diffeomorphism from an open subset $W$ of $M$ onto an open subset $V$ of $N$, we say that $\left.v\right|_{V}$ and $\left.w\right|_{W}$ correspond under $\rho$ when $v(\rho(\zeta))=d \rho_{\zeta}(w(\zeta))$ for all $\zeta \in W$.

Invariance under diffeomorphisms. Let $M \subseteq \mathbb{R}^{n}$ and $N \subseteq \mathbb{R}^{m}$ be differentiable manifolds and let $v: N \rightarrow \mathbb{R}^{n}$ and $w: M \rightarrow \mathbb{R}^{m}$ be tangent vector fields. Assume that $\left.v\right|_{V}$ and $\left.w\right|_{W}$ correspond under some diffeomorphism. Then, if either $v$ is admissible in $V$ or or $w$ is admissible in $W$, so is the other and

$$
\operatorname{deg}(v, V)=\operatorname{deg}(w, W) .
$$

Remark 2.1. Let $W \subseteq M$ be open and relatively compact. If $w: M \rightarrow \mathbb{R}^{m}$ is such that $w(\zeta) \neq 0$ on the boundary $\operatorname{Fr}(W)$ of $W$, then $(w, W)$ is admissible. Let $\varepsilon=\min _{\zeta \in \operatorname{Fr}(W)}|w(\zeta)|$. Then, for any $v: M \rightarrow \mathbb{R}^{m}$ such 
that $\max _{\zeta \in \operatorname{Fr}(W)}|w(\zeta)-v(\zeta)|<\varepsilon$, we have that $(v, W)$ is admissible and that the homotopy $h: M \times[0,1] \rightarrow \mathbb{R}^{m}$ given by

$$
h(\zeta, \lambda)=\lambda w(\zeta)+(1-\lambda) v(\zeta)
$$

is admissible in $W$. Hence, by the Homotopy Invariance Property,

$$
\operatorname{deg}(w, W)=\operatorname{deg}(v, W)
$$

The Excision Property allows the introduction of the notion of index of an isolated zero of a tangent vector field. Let $\zeta \in M$ be an isolated zero of $w$. Clearly, $\operatorname{deg}(w, V)$ is well defined for each open $V \subseteq M$ such that $V \cap w^{-1}(0)=$ $\{\zeta\}$. By the Excision Property $\operatorname{deg}(w, V)$ is constant with respect to such $V^{\prime}$ s. This common value of $\operatorname{deg}(w, V)$ is, by definition, the index of $w$ at $\zeta$, and is denoted by $\mathrm{i}(w, \zeta)$. Using this notation, if $(w, W)$ is admissible, by the Additivity Property we get that if all the zeros in $W$ of $w$ are isolated, then

$$
\operatorname{deg}(w, W)=\sum_{\zeta \in w^{-1}(0) \cap W} \mathrm{i}(w, \zeta) .
$$

By formula (2.1) we have that if $\zeta$ is a nondegenerate zero of $w$, then

$$
\mathrm{i}(w, \zeta)=\operatorname{sign} \operatorname{det} d w_{\zeta}
$$

Notice that (2.1) and (2.2) differ in the fact that, in the latter, the zeros of $w$ are not necessarily nondegenerate as they have to be in the former. In fact, in (2.2), $w$ need not be differentiable at its zeros.

\section{Tangent vector fields on implicitly defined manifolds}

Let $\Psi: \mathbb{R} \times M \rightarrow \mathbb{R}^{m}$ be a (time-dependent) tangent vector field on $M \subseteq \mathbb{R}^{m}$, that is a continuous map with the property that $\Psi(t, \zeta) \in T_{\zeta} M$ for each $(t, \zeta) \in \mathbb{R} \times M$. Assume that there is a connected open subset $U$ of $\mathbb{R}^{m}$ and a smooth map $g: U \rightarrow \mathbb{R}^{s}$ with the property that $M=g^{-1}(0)$. Suppose that with an orthogonal transformation, if necessary, one can write $\mathbb{R}^{m}=\mathbb{R}^{k} \times \mathbb{R}^{s}$, in such a way that the partial derivative of $g$ with respect to the second variable, $\partial_{2} g(x, y)$, is invertible for each $(x, y) \in U$. By this we mean that there exists an orthogonal transformation $P$ of $\mathbb{R}^{m}$ such that the above property holds with the map $\tilde{g}=g \circ P: P^{-1}(U) \rightarrow \mathbb{R}^{s}$ in place of $g$, and with the open set $P^{-1}(U)$ replacing $U$.

To illustrate this point consider by way of example $m=3, s=1, U=\mathbb{R}^{3}$, and $g\left(\xi_{1}, \xi_{2}, \xi_{3}\right)=\xi_{2}+\xi_{1} \xi_{3}$ (here $g^{-1}(0)$ is a hyperbolic paraboloid). Setting $\left(x_{1}, x_{2}, y\right)=\left(\xi_{1}, \xi_{3}, \xi_{2}\right)$ and $\tilde{g}\left(x_{1}, x_{2}, y\right)=y+x_{1} x_{2}$, one has that the partial derivative of $\tilde{g}$ with respect to $y$ is 1 .

As previously remarked in the Introduction, the restrictive assumption we impose on $g$ does not imply that $M=g^{-1}(0)$ is globally a graph. Likewise, one should note that in general a manifold may not be representable as the zero set of a function for which the above condition holds, as in the case of the sphere in $\mathbb{R}^{m}$. 
According to the above decomposition of $\mathbb{R}^{m}$ we can write, for any $\xi \in M$, $\xi=(x, y)$ and, for any $t \in \mathbb{R}$

$$
\Psi(t, \xi)=\Psi(t, x, y)=\left(\Psi_{1}(t, x, y), \Psi_{2}(t, x, y)\right) .
$$

Notice that one must have

$$
\Psi_{2}(t, x, y)=-\left(\partial_{2} g(x, y)\right)^{-1} \partial_{1} g(x, y) \Psi_{1}(t, x, y) .
$$

In fact, $\Psi(t, \xi) \in T_{\xi} M$ being equivalent to $\Psi(t, \xi) \in \operatorname{ker} g^{\prime}(x, y)$, one has for each $(t, x, y) \in \mathbb{R} \times M$ that

$$
0=g^{\prime}(x, y) \Psi(t, x, y)=\partial_{1} g(x, y) \Psi_{1}(t, x, y)+\partial_{2} g(x, y) \Psi_{2}(t, x, y),
$$

which implies (3.1); here $g^{\prime}(x, y)$ denotes the Fréchet derivative of $g$ at $(x, y)$.

We now focus on differential equations on $M$ and write them equivalently (in a sense specified below) as DAEs.

Let us consider the following differential equation on $M$ :

$$
\dot{\xi}=\Psi(t, \xi) \text {. }
$$

By a solution of (3.2) we mean a $C^{1}$ curve $\xi: J \rightarrow \mathbb{R}^{m}$, defined on a (nontrivial) interval $J \subseteq \mathbb{R}$, which satisfies the conditions $\xi(t) \in M$ and $\dot{\xi}(t)=\Psi(t, \xi(t))$, identically on $J$. We need the following fact.

Remark 3.1. If $\Psi$ is as above, since $\mathbb{R} \times M$ is a closed subset of the metric space $\mathbb{R} \times U$, the well known Tietze's Theorem (see e.g. [2]) implies that there exists an extension $\widetilde{\Psi}: \mathbb{R} \times U \rightarrow \mathbb{R}^{m}$ of $\Psi$.

Consider also the 'extended' equation on the neighborhood $U$ of $M$ in $\mathbb{R}^{m}$ :

$$
\dot{\eta}=\widetilde{\Psi}(t, \eta)
$$

where $\widetilde{\Psi}$ is any extension of $\Psi$ as in Remark 3.1. Observe that the solutions of (3.2) are also solutions of (3.3); conversely, the solutions of (3.3) that meet $M$ do actually lie on $M$ and thus are solutions of (3.2).

Equation (3.3) can be conveniently written, setting $\eta=(x, y)$, as the following system:

$$
\left\{\begin{array}{l}
\dot{x}=\Psi_{1}(t, x, y), \\
\dot{y}=\Psi_{2}(t, x, y)
\end{array}\right.
$$

where, for the sake of simplicity, $\widetilde{\Psi}$ has been replaced by $\Psi$.

We claim that (3.2) is equivalent to the following DAE:

$$
\left\{\begin{array}{l}
\dot{x}=\Psi_{1}(t, x, y), \\
g(x, y)=0
\end{array}\right.
$$

Here by a solution of (3.5) we mean a pair of $C^{1}$ functions $x: J \rightarrow \mathbb{R}^{k}$ and $y: J \rightarrow \mathbb{R}^{s}, J$ an interval, with the property that $\dot{x}(t)=\Psi_{1}(t, x(t), y(t))$ and $g(x(t), y(t))=0$ for all $t \in J$.

To prove the claim, let $x: J \rightarrow \mathbb{R}^{k}$ and $y: J \rightarrow \mathbb{R}^{s}$ be $C^{1}$ maps defined on an interval $J$ with the property that $t \mapsto \xi(t)=(x(t), y(t))$ is a solution of (3.2). Then, for all $t \in J, \dot{x}(t)=\Psi_{1}(t, x(t), y(t))$ and, since $(x(t), y(t)) \in M$, 
we have $g(x(t), y(t))=0$. Conversely, if $t \mapsto(x(t), y(t))$ is a solution of (3.5) then, differentiating $g(x(t), y(t))=0$ at any $t \in J$, one gets

$$
\partial_{1} g(x(t), y(t)) \dot{x}(t)+\partial_{2} g(x(t), y(t)) \dot{y}(t)=0 .
$$

So that

$$
\begin{aligned}
\dot{y}(t) & =-\left(\partial_{2} g(x(t), y(t))\right)^{-1} \partial_{1} g(x(t), y(t)) \dot{x}(t) \\
& =-\left(\partial_{2} g(x(t), y(t))\right)^{-1} \partial_{1} g(x(t), y(t)) \Psi_{1}(t, x(t), y(t)) .
\end{aligned}
$$

Taking into account (3.1) and the fact that the solution meets $M$, we have the claim.

\section{Computation of the degree}

As in the previous section, let $M \subseteq \mathbb{R}^{m}$ be a differentiable manifold that is globally defined as a zero set of a suitable map $g: U \rightarrow \mathbb{R}^{s}, U \subseteq \mathbb{R}^{m}$. Here we give a formula for the degree of tangents vector fields on $M$ in terms of (potentially easier to compute) degree of appropriate vector fields on $U$. The main result of this section is Theorem 4.1 below, which is equivalent to a result of [11]. Here we provide a simplified proof.

Throughout this section $\varphi: M \rightarrow \mathbb{R}^{m}$ will be a continuous tangent vector field on M. As in Remark 3.1, Tietze's Theorem implies that there exists an extension $\widetilde{\varphi}: U \rightarrow \mathbb{R}^{m}$ of $\varphi$. Thus, it is not restrictive to assume, as we sometimes do, that the given tangent vector fields are actually defined on a convenient neighborhood of the manifold $M$. In fact, although an arbitrary extension of $\varphi$ may have many zeros outside $M$, we are interested in the degree of $\varphi$ on $M$ which only takes into account those zeros of $\varphi$ that lie on $M$.

Theorem 4.1. Let $U \subseteq \mathbb{R}^{k} \times \mathbb{R}^{s}$ be open and connected, let $g: U \rightarrow \mathbb{R}^{s}$ be a smooth function such that $\partial_{2} g(x, y)$ is nonsingular for any $(x, y) \in U$ and let $M=g^{-1}(0)$. Assume that $\varphi: M \rightarrow \mathbb{R}^{k} \times \mathbb{R}^{s}$ is a continuous tangent vector field on $M$, and let $\widetilde{\varphi}_{1}$ be the projection on $\mathbb{R}^{k}$ of an arbitrary continuous extension $\widetilde{\varphi}$ of $\varphi$ to $U$. Define $\mathcal{F}: U \rightarrow \mathbb{R}^{k} \times \mathbb{R}^{s}$ by $\mathcal{F}(x, y)=\left(\widetilde{\varphi}_{1}(x, y), g(x, y)\right)$. Then, $\mathcal{F}$ is admissible in $U$ if and only if so is $\varphi$ in $M$, and

$$
\operatorname{deg}(\varphi, M)=\mathfrak{s} \operatorname{deg}(\mathcal{F}, U),
$$

where $\mathfrak{s}$ is the constant sign of $\operatorname{det} \partial_{2} g(x, y)$ for all $(x, y) \in U$.

Before we provide the proof of Theorem 4.1, we consider a special case. Observe that a point $(p, q) \in M$ is a zero of $\varphi$ if and only if it is a zero of $\mathcal{F}$.

Lemma 4.2. Let $U, \mathfrak{s}, \varphi$ be as in Theorem 4.1. Assume that $\varphi$ is $C^{1}$ and let $\widetilde{\varphi}=\left(\widetilde{\varphi}_{1}, \widetilde{\varphi}_{2}\right)$ be a $C^{1}$ extension of $\varphi$ to $U$. Let $\mathcal{F}: U \rightarrow \mathbb{R}^{k} \times \mathbb{R}^{s}$ be given by $\mathcal{F}(x, y)=\left(\widetilde{\varphi}_{1}(x, y), g(x, y)\right)$, as in Theorem 4.1 , and suppose that all the zeros of $\mathcal{F}$ are nondegenerate. Then,

$$
\operatorname{deg}(\varphi, M)=\mathfrak{s} \operatorname{deg}(\mathcal{F}, U)
$$


Proof. Observe that since the zeros of $\mathcal{F}$ are nondegenerate, they are also isolated. This implies that the zeros of $\varphi$ are isolated as well. Let $(p, q)$ be a zero of $\mathcal{F}$. As a first step, we will show that

$$
\mathrm{i}(\varphi,(p, q))=\mathfrak{s} \operatorname{sign} \operatorname{det} d \mathcal{F}_{(p, q)} .
$$

Since $\operatorname{det} \partial_{2} g(p, q) \neq 0$, the so-called generalized Gauss algorithm (see e.g. [7]) yields

$$
\begin{aligned}
\operatorname{det} d \mathcal{F}_{(p, q)} & =\operatorname{det}\left(\begin{array}{ll}
\partial_{1} \widetilde{\varphi}_{1}(p, q) & \partial_{2} \widetilde{\varphi}_{1}(p, q) \\
\partial_{1} g(p, q) & \partial_{2} g(p, q)
\end{array}\right) \\
& =\operatorname{det} \partial_{2} g(p, q) \cdot \operatorname{det}\left(\partial_{1} \widetilde{\varphi}_{1}(p, q)-\partial_{2} \widetilde{\varphi}_{1}(p, q)\left(\partial_{2} g(p, q)\right)^{-1} \partial_{1} g(p, q)\right) .3
\end{aligned}
$$

As remarked above, since $(p, q)$ is a nondegenerate zero of $\mathcal{F}$ then it is also isolated, as a zero, of both $\mathcal{F}$ and of $\varphi$ on $M$. Let $B=W \times V$, with $W \subseteq \mathbb{R}^{k}$ and $V \subseteq \mathbb{R}^{s}$ open, be an isolating neighborhood of $(p, q)$ in $\mathbb{R}^{k} \times \mathbb{R}^{s}$ i.e. $B$ is such that $\mathcal{F}(x, y) \neq(0,0)$ for any $(x, y) \in B \backslash\{(p, q)\}$, and $\varphi(x, y) \neq(0,0)$ for any $(x, y) \in B \cap M \backslash\{(p, q)\}$.

Since $\partial_{2} g(p, q)$ is invertible, the implicit function theorem implies that, taking a smaller $W$ if necessary, we can assume that there exists a $C^{1}$ function $\gamma: W \rightarrow \mathbb{R}^{s}$ such that $g(x, \gamma(x))=0$ for any $x \in W$ and $\gamma(W) \subseteq V$. Thus the map $G: x \mapsto(x, \gamma(x))$ is a diffeomorphism of $W$ onto $B \cap M$ whose inverse is the projection $\pi: B \cap M \rightarrow W$ given by $\pi(x, y)=x$.

The property of invariance under diffeomorphisms of the degree of tangent vector fields implies that

$$
\operatorname{deg}(\varphi, B \cap M)=\operatorname{deg}(\pi \circ \varphi \circ G, W) .
$$

Notice that $p$ is an isolated zero of $\pi \circ \varphi \circ G$. Thus, the above relation becomes

$$
\mathrm{i}(\varphi,(p, q))=\mathrm{i}(\pi \circ \varphi \circ G, p)
$$

The differential of $\pi \circ \varphi \circ G$ at $p$ is given by

$$
\partial_{1} \varphi_{1}(p, q)-\partial_{2} \varphi_{1}(p, q)\left(\partial_{2} g(p, q)\right)^{-1} \partial_{1} g(p, q)
$$

(recall that $q=\gamma(p)$ ), which is equal to

$$
\partial_{1} \widetilde{\varphi}_{1}(p, q)-\partial_{2} \widetilde{\varphi}_{1}(p, q)\left(\partial_{2} g(p, q)\right)^{-1} \partial_{1} g(p, q)
$$

because the differential of $\varphi$ at $(p, q)$ coincides with the restriction to $T_{(p, q)} M$ of the differential of $\widetilde{\varphi}$ at the same point. By (4.3) and the fact that $(p, q)$ is a nondegenerate zero of $\mathcal{F}$, it follows that the map in (4.5) is invertible. Therefore, by (4.4) and (2.3), we have

$$
\mathrm{i}(\varphi,(p, q))=\operatorname{sign} \operatorname{det}\left(\partial_{1} \widetilde{\varphi}_{1}(p, q)-\partial_{2} \widetilde{\varphi}_{1}(p, q)\left(\partial_{2} g(p, q)\right)^{-1} \partial_{1} g(p, q)\right) .
$$

Formula (4.2) follows from (4.3) and (4.6).

To complete the proof, let $\left(p_{1}, q_{1}\right), \ldots,\left(p_{n}, q_{n}\right)$ be the zeros of $\mathcal{F}$. Since $\mathfrak{s}$ is constant on the connected set $U$, from (2.2), Lemma 4.2 and (2.1) we have 


$$
\operatorname{deg}(\varphi, M)=\sum_{i=1}^{n} \mathrm{i}\left(\varphi,\left(p_{i}, q_{i}\right)\right)=\sum_{i=1}^{n} \mathfrak{s} \operatorname{sign} \operatorname{det} d \mathcal{F}_{\left(p_{i}, q_{i}\right)}=\mathfrak{s} \operatorname{deg}(\mathcal{F}, U),
$$

that proves the assertion.

Proof of Theorem 4.1. The assertion that $\mathcal{F}$ is admissible in $U$ if and only if so is $\varphi$ in $M$ follows from the identity

$$
\{(p, q) \in M: \varphi(p, q)=0\}=\{(p, q) \in U: \mathcal{F}(p, q)=0\},
$$

which can be deduced from the definition of $\mathcal{F}$ and the fact that, according to $(3.1)$, the projection $\varphi_{2}(x, y)$ of $\varphi(x, y)=\left(\varphi_{1}(x, y), \varphi_{2}(x, y)\right)$, at $(x, y) \in M$ onto $\mathbb{R}^{s}$, is given by

$$
-\left(\partial_{2} g(x, y)\right)^{-1} \partial_{1} g(x, y) \varphi_{1}(x, y) .
$$

Assume now that $\mathcal{F}$ is admissible in $U$. Let $V$ be an open and bounded subset of $U$ with the property that the closure $\bar{V}$ of $V$ is contained in $U$ and that $\mathcal{F}^{-1}(0,0) \subseteq V$. Clearly, $\varphi^{-1}(0,0) \cap M$ is contained in $V$ as well and, by the excision property of the degree of a vector field, we get

$$
\operatorname{deg}(\mathcal{F}, U)=\operatorname{deg}(\mathcal{F}, V), \quad \operatorname{deg}(\varphi, M)=\operatorname{deg}(\varphi, V \cap M) .
$$

Therefore, it is sufficient to prove that

$$
\operatorname{deg}(\varphi, V \cap M)=\mathfrak{s} \operatorname{deg}(\mathcal{F}, V) .
$$

We shall deduce equation (4.7) from Lemma 4.2 via an approximation procedure. Given $\varepsilon>0$, Sard's Lemma implies that one can find a $C^{1}$ map $\mathcal{F}^{\varepsilon}: U \rightarrow \mathbb{R}^{k} \times \mathbb{R}^{s}, \mathcal{F}^{\varepsilon}=\left(\mathcal{F}_{1}^{\varepsilon}, \mathcal{F}_{2}^{\varepsilon}\right)$, that has $(0,0)$ as a regular value and such that

$$
\max _{(x, y) \in \operatorname{Fr}(V)}\left|\mathcal{F}^{\varepsilon}(x, y)-\mathcal{F}(x, y)\right|<\varepsilon .
$$

Define $\widetilde{\psi^{\varepsilon}}: U \rightarrow \mathbb{R}^{k} \times \mathbb{R}^{s}$ by

$$
\widetilde{\psi}^{\varepsilon}(x, y)=\left(\mathcal{F}_{1}^{\varepsilon}(x, y),-\left(\partial_{2} g(x, y)\right)^{-1} \partial_{1} g(x, y) \mathcal{F}_{1}^{\varepsilon}(x, y)\right),
$$

and denote by $\psi^{\varepsilon}$ the restriction of $\widetilde{\psi}^{\varepsilon}$ to $M$. As in Sect. 3, we see immediately that $\psi^{\varepsilon}$ is a tangent vector field on $M$. Recalling formula (3.1), one has

$$
\begin{aligned}
& \sup _{(x, y) \in \operatorname{Fr}(V \cap M)}\left|\psi^{\varepsilon}(x, y)-\varphi(x, y)\right| \leq \sup _{(x, y) \in \operatorname{Fr}(V \cap M)}\left|\mathcal{F}_{1}^{\varepsilon}(x, y)-\mathcal{F}_{1}(x, y)\right| \\
& \quad+\sup _{(x, y) \in \operatorname{Fr}(V \cap M)}\left|\left(\partial_{2} g(x, y)\right)^{-1} \partial_{1} g(x, y)\left(\mathcal{F}_{1}^{\varepsilon}(x, y)-\mathcal{F}_{1}(x, y)\right)\right| \\
& <\varepsilon\left(1+\sup _{(x, y) \in \operatorname{Fr}(V \cap M)}\left\|\left(\partial_{2} g(x, y)\right)^{-1} \partial_{1} g(x, y)\right\|\right)
\end{aligned}
$$

where $|\cdot|$ denotes, according to the space where applied, the Euclidean norm in $\mathbb{R}^{k}, \mathbb{R}^{s}$ or $\mathbb{R}^{k+s}$, and $\|\cdot\|$ denotes the norm of linear operators from $\mathbb{R}^{k}$ to $\mathbb{R}^{s}$. Thus, by the continuity of the partial derivatives of $g$ and the compactness of $\bar{V} \cap M$, it follows that one can choose $\varepsilon$ so small that

$$
\max _{(x, y) \in \operatorname{Fr}(V)}\left|\mathcal{F}^{\varepsilon}(x, y)-\mathcal{F}(x, y)\right|<\min \{|\mathcal{F}(x, y)|:(x, y) \in \operatorname{Fr}(V)\},
$$


and

$$
\max _{(x, y) \in \operatorname{Fr}(V \cap M)}\left|\psi^{\varepsilon}(x, y)-\varphi(x, y)\right|<\min \{|\varphi(x, y)|:(x, y) \in \operatorname{Fr}(V \cap M)\} .
$$

For such a choice of $\varepsilon$ it is easily checked that $\mathcal{F}^{\varepsilon}$ and $\psi^{\varepsilon}$ are admissibly homotopic to $\mathcal{F}$ on $V$ and to $\varphi$ on $V \cap M$, respectively (compare Remark 2.1). Thus,

$$
\operatorname{deg}\left(\mathcal{F}^{\varepsilon}, V\right)=\operatorname{deg}(\mathcal{F}, V)
$$

and

$$
\operatorname{deg}\left(\psi^{\varepsilon}, V \cap M\right)=\operatorname{deg}(\varphi, V \cap M)
$$

Observe also that because of the assumptions on $g$, any zero of $\mathcal{F}^{\varepsilon}$ is nondegenerate. By Lemma 4.2 it follows that

$$
\operatorname{deg}\left(\psi^{\varepsilon}, V \cap M\right)=\mathfrak{s} \operatorname{deg}\left(\mathcal{F}^{\varepsilon}, V\right) .
$$

Now, Equations (4.9), (4.10) and (4.8) imply (4.7). This completes the proof.

Example 4.3. Let $k=s=1, U=\mathbb{R}^{2}$ and $g(x, y)=x^{3}-y^{3}-3 y$. Consider the tangent vector field on $M=g^{-1}(0)$ given by $\varphi(x, y)=\left(x\left(y^{2}+1\right), x^{3}\right)$. Define $\mathcal{F}: U \rightarrow \mathbb{R}^{2}$ by $\mathcal{F}(x, y)=\left(x\left(y^{2}+1\right), x^{3}-y^{3}-3 y\right)$. From the above theorem one gets immediately that $\operatorname{deg}(\varphi, M)=-1 \cdot \operatorname{deg}(\mathcal{F}, U)=+1$.

Example 4.4. Let $s=1, k=2, U=\mathbb{R}^{3}$ and $g\left(x_{1}, x_{2}, y\right)=x_{1}^{2}-y, \varphi\left(x_{1}, x_{2}, y\right)=$ $\left(x_{1}, 1+x_{2}^{3}, 2 x_{1}^{2}\right)$. Put $\varphi_{1}\left(x_{1}, x_{2}, y\right)=\left(x_{1}, 1+x_{2}^{3}\right)$ and $\varphi_{2}\left(x_{1}, x_{2}, y\right)=2 x_{1}^{2}$. Define $\mathcal{F}\left(x_{1}, x_{2}, y\right)=\left(\varphi_{1}\left(x_{1}, x_{2}, y\right), g\left(x_{1}, x_{2}, y\right)\right)=\left(x_{1}, 1+x_{2}^{3}, x_{1}^{2}-y\right)$. The unique zero of $\mathcal{F}$ is $(0,-1,0)$. From the above theorem one gets that $\operatorname{deg}(f, M)=$ $-1 \cdot \operatorname{deg}(\mathcal{F}, U)=+1$.

Theorem 4.1 and the Additivity Property can be combined to get a formula for the degree of a tangent vector field tangent valid in a slightly more general situation.

Corollary 4.5. Let $U \subseteq \mathbb{R}^{k} \times \mathbb{R}^{s}$ be open, $g: U \rightarrow \mathbb{R}^{s}$ a smooth function having $0 \in \mathbb{R}^{s}$ as a regular value and let $M=g^{-1}(0)$. Assume $\varphi: M \rightarrow \mathbb{R}^{k} \times \mathbb{R}^{s}$ is tangent to $M$ and suppose that there are pairwise disjoint open and connected subsets $U_{1}, \ldots, U_{N}$ of $U$ such that

(1) $\varphi^{-1}(0)$ is compact and contained in $\bigcup_{i=1}^{N} U_{i}$;

(2) $\partial_{2} g(x, y)$ is nonsingular for all $(x, y) \in U_{i}, i=1, \ldots, N$.

$$
\operatorname{deg}(\varphi, M)=\sum_{i=1}^{N} \mathfrak{s}_{i} \operatorname{deg}\left(\mathcal{F}, U_{i}\right)
$$

where $\mathcal{F}: U \rightarrow \mathbb{R}^{k} \times \mathbb{R}^{s}$ is defined as in Theorem 4.1 and $\mathfrak{s}_{i}$ denotes the constant sign of $\operatorname{det} \partial_{2} g(x, y)$ in $U_{i}$, for $i=1, \ldots, N$. 


\section{Applications and examples}

This section is devoted to the study of the set of $T$-periodic solutions of equations (1.2).

Let us introduce some notation. We shall denote by $C_{T}(M)$ the set of the continuous $T$-periodic maps from $\mathbb{R}$ to $M$ with the metric induced by the Banach space $C_{T}\left(\mathbb{R}^{m}\right)$ of the continuous $T$-periodic $\mathbb{R}^{m}$-valued maps (with the standard supremum norm). For the sake of simplicity we make some conventions. We will regard every space as its image in the following diagram of natural inclusions

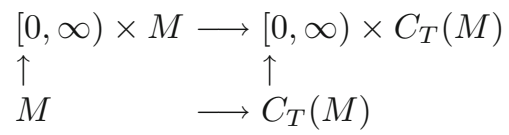

In particular, we will identify $M$ with its image in $C_{T}(M)$ under the embedding which associates to any $\zeta \in M$ the map $\hat{\zeta} \in C_{T}(M)$ constantly equal to $\zeta$. Moreover we will regard $M$ as the slice $\{0\} \times M \subset[0, \infty) \times M$ and, analogously, $C_{T}(M)$ as $\{0\} \times C_{T}(M)$. We point out that the images of the above inclusions are closed.

According to these identifications, if $\Omega$ is an open subset of $[0, \infty) \times$ $C_{T}(M)$, by $\Omega \cap M$ we mean the open subset of $M$ given by all $\zeta \in M$ such that the pair $(0, \hat{\zeta})$ belongs to $\Omega$. If $\mathcal{O}$ is an open subset of $[0, \infty) \times M$, then $\mathcal{O} \cap M$ represents the open set $\{\zeta \in M:(0, \zeta) \in \mathcal{O}\}$.

We say that $(\mu ; x, y) \in[0, \infty) \times C_{T}(M)$ is a solution pair of (1.2a) if $\xi=(x, y)$ satisfies (1.2a) for $\lambda=\mu$; here the pair $(x, y)$ is thought of as a single element of $C_{T}(M)$. Given $\zeta=(p, q) \in M$, a solution pair of the form $(0 ; \hat{p}, \hat{q})$ is called trivial.

Throughout this section $U$ will be an open and connected subset of $\mathbb{R}^{k} \times$ $\mathbb{R}^{s}$. We will always assume that $g: U \rightarrow \mathbb{R}^{s}$ is a smooth function such that $\partial_{2} g(x, y)$ is nonsingular for any $(x, y) \in U$, and $M=g^{-1}(0)$. It will also be convenient, given a continuous tangent vector field, $f: M \rightarrow \mathbb{R}^{k} \times \mathbb{R}^{s}$, to denote by $\tilde{f}$ an arbitrary extension of $f$ to $U$ (as in Remark 3.1 ) and to let $\widetilde{f}_{1}(x, y)$ be the projection of $\widetilde{f}(x, y)$ on $\mathbb{R}^{k}$ for any $(x, y) \in U$.

Theorem 5.1. Let $f: M \rightarrow \mathbb{R}^{k} \times \mathbb{R}^{s}$, and $h: \mathbb{R} \times M \rightarrow \mathbb{R}^{k} \times \mathbb{R}^{s}$ be continuous tangent vector fields, with $h$ of a given period $T>0$ in the first variable. Define $\mathcal{F}: U \rightarrow \mathbb{R}^{k} \times \mathbb{R}^{s}$ by $\mathcal{F}(x, y)=\left(\widetilde{f}_{1}(x, y), g(x, y)\right)$ for any $(x, y) \in U$. Given an open set $\Omega \subseteq[0, \infty) \times C_{T}(M)$, let $\mathcal{O} \subseteq \mathbb{R}^{m}$ be open with the property that $\mathcal{O} \cap M=\Omega \cap M$. Assume that $\operatorname{deg}(\mathcal{F}, \mathcal{O})$ is well defined and nonzero. Then there exists a connected set $\Gamma$ of nontrivial solution pairs for (1.2a) in $\Omega$ whose closure in $\Omega$ meets $f^{-1}(0) \cap \Omega$ and is not compact. In particular, if $\Omega=[0, \infty) \times C_{T}(M)$, then $\Gamma$ is unbounded.

Proof. By Theorem 4.1 we have

$$
|\operatorname{deg}(f, \Omega \cap M)|=|\operatorname{deg}(f, \mathcal{O} \cap M)|=|\operatorname{deg}(\mathcal{F}, \mathcal{O})| .
$$

Thus, $\operatorname{deg}(f, \Omega \cap M) \neq 0$ and the assertion follows from Theorem 3.3 of [6]. 
Example 5.2. Let $s=2, k=1, U=\mathbb{R}^{3}$ and consider $g: \mathbb{R} \times \mathbb{R}^{2} \rightarrow \mathbb{R}^{2}$ given by

$$
g(x, y)=g\left(x ; y_{1}, y_{2}\right)=\left(e^{y_{1}} \cos y_{2}-x, e^{y_{1}} \sin y_{2}+x-1\right) .
$$

where $y=\left(y_{1}, y_{2}\right)$. Clearly, although for each $(x, y) \in \mathbb{R} \times \mathbb{R}^{2}$

$$
\operatorname{det} \partial_{2} g(x, y)=\operatorname{det}\left(\begin{array}{cc}
e^{y_{1}} \cos y_{2} & e^{y_{1}} \sin y_{2} \\
-e^{y_{1}} \sin y_{2} & e^{y_{1}} \cos y_{2}
\end{array}\right)=e^{2 y_{1}}>0
$$

$M=g^{-1}(0)$ is not the graph of a map $x \mapsto y(x)$. Consider the following ODE on $M$ :

$$
\dot{\xi}=f(\xi),
$$

where $\xi=\left(x, y_{1}, y_{2}\right)$ and $f$ is the tangent vector field given by

$$
f\left(x, y_{1}, y_{2}\right)=\left(y_{2}, y_{2}\left(\cos y_{2}+\sin y_{2}\right) e^{-y_{1}},-y_{2}\left(\cos y_{2}-\sin y_{2}\right) e^{-y_{1}}\right) \text {. }
$$

Define $\mathcal{F}\left(x, y_{1}, y_{2}\right)=\left(y_{2}, e^{y_{1}} \cos y_{2}-x, e^{y_{1}} \sin y_{2}+x-1\right)$, for $\left(x, y_{1}, y_{2}\right) \in \mathbb{R}^{3}$. From Theorem 4.1 we get $\operatorname{deg}(f, M)=\operatorname{deg}\left(\mathcal{F}, \mathbb{R}^{3}\right)=-1$.

Clearly $f^{-1}(0)=\{(1,0,0)\}$. Thus, letting $\Omega=[0, \infty) \times C_{T}(M)$ in Theorem 5.1, one has that given any $T$-periodic vector field $h: \mathbb{R} \times \mathbb{R}^{3} \rightarrow \mathbb{R}^{3}$ tangent to $M$ there exists an unbounded connected set $\Gamma$ of nontrivial solution pairs of equation

$$
\dot{\xi}=f(\xi)+\lambda h(t, \xi), \quad \lambda \geq 0,
$$

whose closure in $[0, \infty) \times C_{T}(M)$ meets $\{(0, \hat{\zeta})\}$ where $\hat{\zeta} \in C_{T}(M)$ is the function constantly equal to $(1,0,0)$.

Let us now consider Equation (1.2b). Let $g$ and $h$ be as above, and suppose that $h$ is $T$-periodic in the first variable for a given $T>0$. We want to derive a continuation result for $(1.2 \mathrm{~b})$, analogous to Theorem 5.1 above, following [1]. We say that $(\mu ; x, y) \in[0, \infty) \times C_{T}(U)$ is a solution pair of $(1.2 \mathrm{~b})$ if $\xi=(x, y)$ satisfies (1.2b) for $\lambda=\mu$. Given $\zeta=(p, q) \in M$, a solution pair of the form $(0 ; \hat{p}, \hat{q})$ is called trivial.

Define the 'average wind' vector field $w^{h}$ on $M$ by

$$
w^{h}(\xi)=\frac{1}{T} \int_{0}^{T} h(t, \xi) d t .
$$

The following result concerns Equation (1.2b).

Theorem 5.3. Let $h: \mathbb{R} \times M \rightarrow \mathbb{R}^{k} \times \mathbb{R}^{s}$ be a continuous tangent vector field, of a given period $T>0$ in the first variable. Define $\Phi: U \rightarrow \mathbb{R}^{k} \times \mathbb{R}^{s}$ by $\Phi(x, y)=$ $\left(\widetilde{w}_{1}^{h}(x, y), g(x, y)\right)$ for any $(x, y) \in U$. Given an open set $\Omega \subseteq[0, \infty) \times C_{T}(U)$, let $\mathcal{O} \subset \mathbb{R}^{m}$ be an open subset with the property that $\Omega \cap M=\mathcal{O} \cap M$. Assume that $\operatorname{deg}(\Phi, \mathcal{O})$ is well defined and nonzero. Then there exists a connected set $\Gamma$ of nontrivial solution pairs for $(1.2 \mathrm{~b})$ in $\Omega$ whose closure in $\Omega$ is not compact and meets the set $\left(w^{h}\right)^{-1}(0) \cap \Omega$. In particular, if $\Omega=[0, \infty) \times C_{T}(U)$, then $\Gamma$ is unbounded.

Proof. By Theorem 4.1 we have

$$
\left|\operatorname{deg}\left(w^{h}, \Omega \cap M\right)\right|=\left|\operatorname{deg}\left(w^{h}, \mathcal{O} \cap M\right)\right|=|\operatorname{deg}(\Phi, \mathcal{O})| .
$$


Thus, $\operatorname{deg}\left(w^{h}, \Omega \cap M\right) \neq 0$ and the assertion follows from Theorem 2.2 of [3].

Example 5.4. Let $k=s=1$ and let $U=\mathbb{R}^{2}$. Consider the map

$$
g(x, y)=y^{3}+y-x^{2} .
$$

Clearly, $\partial_{2} g(x, y)=3 y^{2}+1>0$ for all $\xi=(x, y) \in \mathbb{R}^{2}$. Consider the following ODE on $M=g^{-1}(0)$ :

$$
\dot{\xi}=\lambda h(t, \xi), \quad \lambda \geq 0
$$

where the $2 \pi$-periodic tangent vector field $h$ is given by

$$
h(t, x, y)=\left(x+y+\sin t, \frac{2 x(x+y+\sin t)}{3 y^{2}+1}\right) .
$$

Define

$$
\Phi(x, y)=\left(x+y, y^{3}+y-x^{2}\right) .
$$

Observe that $\Phi^{-1}(0,0)=\{(0,0)\}$ and $\operatorname{deg}\left(\Phi, \mathbb{R}^{2}\right)=1$, so that Theorem 5.3 applies with $\Omega=[0, \infty) \times C_{T}\left(\mathbb{R}^{2}\right)$ yielding the existence of an unbounded branch of solution pairs of (5.1).

\subsection{Applications to a class of differential-algebraic equations (DAEs)}

Let us now consider applications to semi-explicit DAEs of the form (1.3). As above, we will consider the case when $U \subseteq \mathbb{R}^{k} \times \mathbb{R}^{s}$ is open and connected and $g: U \rightarrow \mathbb{R}^{s}$ is smooth and such that $\partial_{2} g(x, y)$ is invertible for all $(x, y) \in U$. For equations (1.3) we will write explicitly the tangent vector fields $f$ and $h$ that carry out the equivalence of of (1.3a) with (1.2a) and of (1.3b) with $(1.2 \mathrm{~b})$, respectively. The argument is parallel to that of Sect. 3 .

Let us consider equations on an open connected set $U \subseteq \mathbb{R}^{k} \times \mathbb{R}^{s}$ of the following form:

$$
\left\{\begin{array}{l}
\dot{x}=F(t, x, y) \\
g(x, y)=0
\end{array}\right.
$$

where $F: \mathbb{R} \times U \rightarrow \mathbb{R}^{k}$ is continuous and $g: U \rightarrow \mathbb{R}^{s}$ is smooth and such that $\partial_{2} g(x, y)$ is invertible for all $(x, y) \in U$. It is well known (compare [9, §4.5]) and easy to see that in this situation, equation (5.2) induces a tangent vector field $\Psi$ on $M$, that is, it gives rise to an ODE on $M=g^{-1}(0) \subseteq \mathbb{R}^{k} \times \mathbb{R}^{s}$. In fact, one can see that setting

$$
\Psi(t ; x, y)=\left(F(t, x, y),-\left(\partial_{2} g(x, y)\right)^{-1} \partial_{1} g(x, y) F(t, x, y)\right),
$$

equation (5.2) is equivalent to the ODE

$$
\dot{\xi}=\Psi(t, \xi)
$$

on $M$, where $\xi=(x, y)$.

Given continuous maps $\gamma: U \rightarrow \mathbb{R}^{k}$ and $\sigma: \mathbb{R} \times U \rightarrow \mathbb{R}^{k}$, define the tangent vector fields $f: M \rightarrow \mathbb{R}^{k} \times \mathbb{R}^{s}$ and $h: M \rightarrow \mathbb{R}^{k} \times \mathbb{R}^{s}$ on $M$ by

$$
f(x, y)=\left(\gamma(x, y),-\left(\partial_{2} g(x, y)\right)^{-1} \partial_{1} g(x, y) \gamma(x, y)\right),
$$


and

$$
h(t, x, y)=\left(\sigma(t, x, y),-\left(\partial_{2} g(x, y)\right)^{-1} \partial_{1} g(x, y) \sigma(t, x, y)\right) .
$$

Recalling formula (3.1), the above argument shows that (1.3a) and (1.3b) are equivalent to (1.2a) and (1.2b), respectively. Also, if $\sigma$ is $T$-periodic in the first variable, so is $h$.

We are going to use this equivalence to deduce some of the results of [1] and of [1] for equations of the form (1.3a) and (1.3b), respectively. Let us begin with equations of the form (1.3a).

We need to introduce some further notation. We say that $(\mu ; x, y) \in$ $[0, \infty) \times C_{T}(U)$ is a solution pair of (1.3a) if $(x, y)$ satisfies (1.3a) for $\lambda=\mu$. It is convenient, given any $(p, q) \in \mathbb{R}^{k} \times \mathbb{R}^{s}$, to denote by $(\hat{p}, \hat{q})$ the map in $C_{T}\left(\mathbb{R}^{k} \times \mathbb{R}^{s}\right)$ that is constantly equal to $(p, q)$. A solution pair of the form $(0 ; \hat{p}, \hat{q})$ is called trivial.

Let $\mathcal{F}: U \rightarrow \mathbb{R}^{k} \times \mathbb{R}^{s}$ be given by $\mathcal{F}(x, y)=(\gamma(x, y), g(x, y))$. As one immediately checks, $(\hat{p}, \hat{q})$ is a constant solution of $(1.3 \mathrm{a})$ corresponding to $\lambda=0$ if and only if $\mathcal{F}(p, q)=(0,0)$. Thus, with this notation, the set of trivial solution pairs of $(1.3 \mathrm{a})$ can be written as

$$
\left\{(0 ; \hat{p}, \hat{q}) \in[0, \infty) \times C_{T}(U): \mathcal{F}(p, q)=(0,0)\right\} .
$$

Given $\Omega \subseteq[0, \infty) \times C_{T}(U)$, with $U \cap \Omega$ we denote the set of points of $U$ that, regarded as constant functions, lie in $\Omega$. Namely,

$$
U \cap \Omega=\{(p, q) \in U:(0 ; \hat{p}, \hat{q}) \in \Omega\} .
$$

We are now ready to state and prove a result concerning the $T$-periodic solutions of $(1.3 \mathrm{a})$.

Theorem 5.5. ([11]) Let $U \subseteq \mathbb{R}^{k} \times \mathbb{R}^{s}$ be open and connected. Let $g: U \rightarrow$ $\mathbb{R}^{s}, \gamma: U \rightarrow \mathbb{R}^{k}, \sigma: \mathbb{R} \times U \rightarrow \mathbb{R}^{k}$ and $T>0$ be such that $\gamma$ and $\sigma$ are continuous, $\sigma$ being $T$-periodic in the first variable, and $g$ is smooth with $\partial_{2} g(x, y)$ invertible for all $(x, y) \in U$. Let also $\mathcal{F}(x, y)=(\gamma(x, y), g(x, y))$. Given $\Omega \subseteq$ $[0, \infty) \times C_{T}(U)$ open, assume $\operatorname{deg}(\mathcal{F}, U \cap \Omega)$ is well-defined and nonzero. Then, there exists a connected set $\Gamma$ of nontrivial solution pairs of (1.3a) whose closure in $\Omega$ is not compact and meets the set $\{(0, \hat{p}, \hat{q}) \in \Omega: \mathcal{F}(p, q)=(0,0)\}$.

Proof. Let $f: M \rightarrow \mathbb{R}^{k} \times \mathbb{R}^{s}$ and $h: M \rightarrow \mathbb{R}^{k} \times \mathbb{R}^{s}$ be given by (5.3) and (5.4), respectively. Then, as remarked above (1.3a) is equivalent to (1.2a) on $M=g^{-1}(0)$. This equivalence implies that each pair $(\lambda ; x, y)$ can be thought as a solution pair of (1.2a) and vice versa. The assertion follows from Theorem 5.1.

The following example could be treated with classical methods because of the asymptotic behavior at infinity of the implicit function. Nevertheless, we choose to include it here as an illustration of our results.

Example 5.6. Consider the second order DAE

$$
\left\{\begin{array}{l}
\ddot{x}=-y-\alpha \dot{x}+\lambda \sigma(t, x, \dot{x}), \quad \lambda \geq 0 \\
y^{3}+y-x^{5}-x=0
\end{array}\right.
$$


that represents the motion with friction $-\alpha \dot{x}, \alpha>0$, of a unit mass particle constrained to the real axis and attached to the origin with an initially 'stiff' nonlinear spring (such that the displacement $x$ and the reaction force $-y$ are related implicitly by $\left.y^{3}+y=x^{5}+x\right)$, and acted on by a $T$-periodic force $\sigma$ depending on position and velocity. Let us rewrite equivalently (5.5) as a first order DAE of the form (1.3a).

$$
\left\{\begin{array}{l}
\dot{x}_{1}=x_{2} \\
\dot{x}_{2}=-y-\alpha x_{2}+\lambda \sigma\left(t ; x_{1}, x_{2}\right), \quad \lambda \geq 0 \\
y^{3}+y-x_{1}^{5}-x_{1}=0 .
\end{array}\right.
$$

Take $U=\mathbb{R}^{2} \times \mathbb{R}$ and define $\mathcal{F}\left(x_{1}, x_{2}, y\right)=\left(x_{2},-\alpha x_{2}-y, y^{3}+y-x_{1}^{5}-x_{1}\right)$. Since $\operatorname{deg}(\mathcal{F}, U)=1$, Theorem 5.5 yields an unbounded connected set $\Gamma$ of nontrivial solution pairs of (5.6) emanating from the solution constantly equal to $(0,0,0)$. Clearly, each element of $\Gamma$ corresponds to a nonconstant $T$-periodic solution of (5.5). In fact, an energy argument shows that (5.5) has only constant periodic solutions for $\lambda=0$. Thus, $\Gamma$ has no intersection with the slice $\{0\} \times C_{T}(U)$.

In a similar way we deduce a continuation result for equation (1.3b) from Theorem 5.3 above. In the following we will say that $(\mu ; x, y) \in[0, \infty) \times C_{T}(U)$ is a solution pair of $(1.3 \mathrm{~b})$ if $(x, y)$ satisfies $(1.3 \mathrm{~b})$ for $\lambda=\mu$. A solution pair of the form $(0 ; \hat{p}, \hat{q})$ will be called trivial.

Theorem 5.7. ([1]) Let $U \subseteq \mathbb{R}^{k} \times \mathbb{R}^{s}$ be open and connected. Let $g: U \rightarrow \mathbb{R}^{s}$ be smooth with $\partial_{2} g(x, y)$ invertible for all $(x, y) \in U$, and $\sigma: \mathbb{R} \times U \rightarrow \mathbb{R}^{k}$ continuous and T-periodic in the first variable. Let also $\Phi: U \rightarrow \mathbb{R}^{k} \times \mathbb{R}^{s}$ be given by $\Phi(x, y)=(\Sigma(x, y), g(x, y))$, where

$$
\Sigma(x, y)=\frac{1}{T} \int_{0}^{T} \sigma(t, x, y) d t .
$$

Given $\Omega \subseteq[0, \infty) \times C_{T}(U)$ open, assume that $\operatorname{deg}(\Phi, U \cap \Omega)$ is well-defined and nonzero. Then, there exists a connected set $\Gamma$ of nontrivial solution pairs of $(1.3 \mathrm{~b})$ whose closure in $\Omega$ is not compact and meets the set $\{(0, \hat{p}, \hat{q}) \in \Omega$ : $\Phi(p, q)=(0,0)\}$.

Proof. Let $h: M \rightarrow \mathbb{R}^{k} \times \mathbb{R}^{s}$ be the tangent vector field on $M$ given by (5.4). Then, equation (1.3b) is equivalent to $(1.2 \mathrm{~b})$ on $M=g^{-1}(0)$, and the assertion follows from Theorem 5.3.

Example 5.8. Consider the following DAE in the form (1.3b) with $T=2 \pi$ :

$$
\left\{\begin{array}{l}
\dot{x}_{1}=\lambda\left(y_{2}+\cos t\right) \\
\dot{x}_{2}=\lambda\left(y_{1}-2 \cos ^{2} t\right) \\
x_{1}-y_{1} \cos y_{2}=0 \\
x_{2}-y_{1} \sin y_{2}=0
\end{array} \quad y_{1}>0\right.
$$

Here, $s=2, k=2, U=\left\{\left(x_{1}, x_{2} ; y_{1}, y_{2}\right) \in \mathbb{R}^{2} \times \mathbb{R}^{2}, y_{1}>0\right\}$. Let $g: U \rightarrow \mathbb{R}^{2}$ be given by

$$
g(x, y)=g\left(x_{1}, x_{2} ; y_{1}, y_{2}\right)=\left(x_{1}-y_{1} \cos y_{2}, x_{2}-y_{1} \sin y_{2}\right) .
$$


where $x=\left(x_{1}, x_{2}\right)$ and $y=\left(y_{1}, y_{2}\right)$. One has,

$$
\operatorname{det} \partial_{2} g(x, y)=\operatorname{det}\left(\begin{array}{l}
\cos y_{2} y_{1} \sin y_{2} \\
\sin y_{2}-y_{1} \cos y_{2}
\end{array}\right)=y_{1}>0 \text {. }
$$

Clearly, the 2-dimensional manifold $M$ cannot be written as the graph of a function $\left(x_{1}, x_{2}\right) \mapsto\left(y_{1}\left(x_{1}, x_{2}\right), y_{2}\left(x_{1}, x_{2}\right)\right)$. Let $\Phi: U \rightarrow \mathbb{R}^{4}$ be given by

$$
\Phi(x, y)=\Phi\left(x_{1}, x_{2} ; y_{1}, y_{2}\right)=\left(y_{2}, y_{1}-1, x_{1}-y_{1} \cos y_{2}, x_{2}-y_{1} \sin y_{2}\right) .
$$

A straightforward computation shows that $\Phi^{-1}(0)=\{(1,0,1,0)\}$ and that $\operatorname{deg}(\Phi, U)=-1$. Then, Theorem 5.7 applies with $\Omega=[0, \infty) \times C_{T}(U)$, yielding the existence of an unbounded branch of nontrivial solution pairs of (5.7).

\section{References}

[1] Calamai, A.: Branches of harmonic solutions for a class of periodic differentialalgebraic equations. Commun. Appl. Anal. (to appear)

[2] Dugundij, J.: Topology. Allyn and Bacon Series in Advanced Mathematics. Allyn and Bacon, Boston (1966)

[3] Furi, M., Pera, M.P.: Carathéodory periodic perturbations of the zero vector field on manifolds. Topol. Methods Nonlinear Anal. 10, 79-92 (1997)

[4] Furi, M., Pera, M.P., Spadini, M.: Forced oscillations on manifolds and multiplicity results for periodically perturbed autonomous systems. J. Comput. Appl. Math. 113, 241-254 (2000)

[5] Furi, M., Pera, M.P., Spadini, M.: The fixed point index of the Poincaré operator on differentiable manifolds. In: Brown, R.F., Furi, M., Górniewicz, L., Jiang, B. (eds.) Handbook of Topological Fixed Point Theory. Springer (2005)

[6] Furi, M., Spadini, M.: On the set of harmonic solutions of periodically perturbed autonomous differential equations on manifolds. Nonlinear Anal. TMA 29(8), 963-970 (1997)

[7] Gantmacher, F.R.: Théorie des matrices (tome 1). Collection Univ. de Mathématiques, Dunod, Paris (1966)

[8] Hirsch, M.W.: Differential topology. Graduate Texts in Math., vol. 33. Springer, Berlin (1976)

[9] Kunkel, P., Mehrmann, V.: Differential algebraic equations, analysis and numerical solutions. In: EMS Textbooks in Mathematics. European Mathematical Society, Zürich (2006)

[10] Milnor, J.W.: Topology from the Differentiable Viewpoint. University Press of Virginia, Charlottesville (1965)

[11] Spadini, M.: A note on topological methods for a class of differential-algebraic equations. Nonlinear Anal. 73, 1065-1076 (2010) 


\author{
Alessandro Calamai \\ Dipartimento di Scienze Matematiche \\ Università Politecnica delle Marche \\ Via Brecce Bianche \\ 60131 Ancona \\ Italy \\ e-mail: calamai@dipmat.univpm.it \\ Marco Spadini \\ Dipartimento di Matematica Applicata \\ Università di Firenze \\ Via Santa Marta 3 \\ 50139 Florence \\ Italy
}

Received: 8 February 2011.

Accepted: 8 September 2011. 